\title{
Ultrasonic Stress Evaluation through Thickness of a Stainless Steel Pressure Vessel
}

Abstract

This paper investigates ultrasonic method in stress measurement through thickness of a pressure vessel. Longitudinal critically refracted $\left(L_{C R}\right)$ waves are employed to measure the welding residual stresses in a vessel constructed from austenitic stainless steel 304L. The acoustoelastic constant is measured through a hydro test to keep the pressure vessel intact. Hoop and axial residual stresses are evaluated by using different frequency range of ultrasonic transducers. The welding processes of vessel shell and caps are simulated by a 3D finite element (FE) model which is validated by hole-drilling method. The residual stresses calculated by FE simulation are then compared with those obtained from the ultrasonic measurement while a good agreement is observed. It is demonstrated that the residual stresses through thickness of the stainless steel pressure vessel can be evaluated by combining $F E$ and $L_{C R}$ method (known as $F E L_{C R}$ method).

Keywords: Finite Element Welding Simulation; Pressure Vessel; Ultrasonic Stress Measurement; Through Thickness Stress; Acoustoelastic Constant.

\section{INTRODUCTION}

The engineering properties of the structural components, particularly fatigue life, deformation, dimensional stability, corrosion resistance, and brittle fracture can be significantly influenced by residual stresses. Residual stresses can be defined as the stresses that remain within a material after manufacturing, in the absence of external loads or thermal gradients. Welding is an essential production process in the industry which generates residual stresses at a notable level. The welding residual stresses are formed in the structure as the result of differential contractions during the weld cooling. Ultrasonic method, known also as longitudinal critically refracted $\left(L_{C R}\right)$ wave techniques, is not limited by the material type and can be employed for stresses measurements on thick samples. The technique is based on the acoustoelastic effect, according to which the velocity of elastic wave propagated in solids depends on the mechanical stress.

Ultrasonic stress evaluation was discussed by Thompson, et al. [1] and Schneider [2]. Applications of the $L_{C R}$ technique were given in a number of studies, remarkably Santos and Bray [3, 4] and Bray and Chance [5]. Tanala et al. [6] explained a combination of subsurface longitudinal and Rayleigh waves to determine the welding stresses near surface. Employing the subsurface longitudinal waves in rail stress measurement has been studied by Szelazek [7].

Pressure vessels, piping and tanks are important engineering equipments which contain various types of fluids at the range of temperatures and pressures. Residual stresses were ultrasonically measured in a pressure vessel by Bray [8]. He constructed a pressure vessel from A106 carbon steel to demonstrate the $L_{C R}$ ultrasonic technique for indicating changes in wall and weld stress. However, he did not completely measure the hoop and axial residual stresses of the pressure vessel. Since stainless steel pressure vessels are widely utilized in the industry, the residual stress measurement of a pressure vessel constructed from $304 \mathrm{~L}$ stainless steel is investigated in this study. The ultrasonic measurements are verified with the values of stresses measured by hole-drilling method. However, the hole-drilling and the other common non-destructive methods for stress measurement (like X-ray diffraction, Barkhausen Noise and ...) are considered as the surface methods [9]. In this study, residual stresses through the thickness of pressure vessel are evaluated by four different series ( $1 \mathrm{MHz}, 2 \mathrm{MHz}, 4 \mathrm{MHz}$ and $5 \mathrm{MHz}$ ) of ultrasonic transducers. Furthermore, a 3D finite element model of pressure vessel is used to verify the ultrasonic results through the thickness. The combination of finite element and $L_{C R}$ method (known as $F E L_{C R}$ ) is employed to evaluate the pressure vessel residual stresses through the thickness. 


\section{THEORETICAL BACKGROUND}

\section{1. $L_{C R}$ method}

Different ultrasonic configurations can be utilized for residual stresses measurements by $L_{C R}$ waves. Generally, waves are launched by a transmitter transducer, propagate through a region of the material, and are detected by a receiver transducer, as show in Fig. 1 . The residual stress in a subsurface layer is determined while the depth of layer is related to the ultrasonic wave-length, often exceeding a few millimetres.

The relation between measured travel-time change of $L_{C R}$ wave and the corresponding uniaxial stress is derived by Egle and Bray [10] to be:

$$
\Delta \sigma=\frac{E}{L t_{0}}\left(t-t_{0}-\Delta t_{T}\right)
$$

, where $\Delta \sigma$ is stress change, $E$ is the elastic modulus and $L$ is the acoustoelastic constant for longitudinal waves propagating in the direction of the applied stress field. Also, $t$ is the experimental travel-time which would be measured on the welded structure that is being evaluated; $t_{0}$ is travel-time for a homogeneous, isotropic, stressfree sample at the room temperature and $\Delta t_{T}$ is temperature difference effect between the measurement temperature and room temperature on the travel-time. With knowledge of the weld induced change in travel-time and the measured acoustoelastic constant, the stress change produced by the weld may be calculated.

\subsection{Finite Element Welding Simulation}

Numerical simulation of welding residual stresses needs to accurately take into account the interactions between heat transfer, metallurgical transformations and mechanical fields. The phenomena involved in the heat input such as arc, material interactions, as well as, fluid dynamics in the weld pool are not precisely described. From the thermo-mechanical point of view, the heat input can be seen as a volumetric or surfaced energy distribution, and the fluid flow effect, which leads to homogenized temperature in the molten area, can be simply taken into account by increasing the thermal conductivity over the melting temperature. As no metallurgical transformation occurs in the austenitic stainless steel (304L), the detailed modelling of the melting is not considered. Heat transfers in solids are described by the heat equation as following:

$$
\rho \frac{d H}{d t}-\operatorname{div}(k \nabla T)-Q=0
$$

$$
k \nabla T . n=q(T, t) \quad \text { on } \partial \Omega_{q}
$$

$$
T=T_{p}(t) \quad \text { on } \partial \Omega_{t}
$$

, where $\rho, H, K$ and $T$ are density, enthalpy, thermal conductivity and temperature respectively. $Q$ represents the internal heat source. In Eq. (3), $n$ is the outward normal vector of domain $\partial \Omega$ and $q$ the heat flux density that can rely on temperature and time to model convective heat exchanges on the surface and $T_{p}$ is a prescribed temperature. In the present study, the double ellipsoid heat source pattern proposed by Goldak et al. [11] is used. The Goldak equations for the front and rear heat source are expressed by the Eq. 5 and Eq. 6 respectively:

$$
\begin{aligned}
& q_{f}(x, y, z)=\frac{6 \sqrt{3} f_{f} Q}{a b c_{f} \pi^{3 / 2}} e^{\left(-3 x^{2} / a^{2}\right)} e^{\left(-3 y^{2} / b^{2}\right)} e^{\left(-3 z^{2} / c^{2}\right)} \\
& q_{r}(x, y, z)=\frac{6 \sqrt{3} f_{r} Q}{a b c_{r} \pi^{3 / 2}} e^{\left(-3 x^{2} / a^{2}\right)} e^{\left(-3 y^{2} / b^{2}\right)} e^{\left(-3 z^{2} / c^{2}\right)}
\end{aligned}
$$

, where $x, y$ and $z$ are the local coordinates of the double ellipsoid model aligned with the welded vessel; $f_{f}$ and $f_{r}$ are parameters which give the fraction of the heat deposited in the front and rear parts, respectively. $Q$ is the welding 
energy which is calculated by knowing the welding current and voltage and considering the arc efficiency. The parameter $a$ is one-half the width of melted zone; $b$ is depth of the melted zone; $c_{f}$ and $c_{r}$ are the front and behind section dimensions of the heat source respectively. In this study, the parameter $a$ and $b$ are experimentally measured for all the welding passes of the main and cap welds. The front section dimension of the heat source $\left(c_{f}\right)$ is assumed equal to $a$ and the rear section $\left(c_{r}\right)$ is equal to $4 \times a$. The relations $2 /\left(1+c_{r} / c_{f}\right)$ and $2 /\left(1+c_{f} / c_{r}\right)$ are used to calculate the $f_{f}$ and $f_{r}$ respectively. The moving heat source is modelled by a user subroutine in the ANSYS software.

In the thermal analysis, the boundary conditions include the radiation and convection to the environment from all of the vessel surfaces except the area under the heat source. Radiation losses are significant for higher temperatures near the weld zone, while convection losses are higher for lower temperatures nodes away from the weld. A user subroutine is employed to simulate the combined thermal boundary condition while the heat convection coefficient is assumed $8 \mathrm{~W} / \mathrm{m}^{2} \mathrm{~K}$. The mechanical boundary conditions are the structural constraints which are set on the FE model according to the experimental constraints employed during the welding process of the pressure vessel.

The mechanical analysis is based on the usual equations of the static equilibrium. As the plastic dissipation is neglected in the thermal analysis, thermal and mechanical analysis can be treated separately. The temperature fields computed by the thermal analysis, is used in the mechanical analysis. The materials are supposed to follow an elastic-plastic behavior with isotropic hardening. The material parameters, young's modulus, poisson's ratio, yield stress, strain hardening and heat expansion coefficient are temperature dependent.

Material modelling has always been a serious issue in the simulation of welding because of the scarcity of material data at elevated temperatures. Some simplifications and approximations are typically introduced to cope with this problem. These simplifications are necessary because of both lack of data and numerical problems when trying to model the actual high-temperature behaviour of the material [12]. The material properties of $304 \mathrm{~L}$ stainless used in the finite element analysis is extracted from X.K.Zhu et al. [13].

The problem is formulated as a successively coupled thermal stress analysis. First, a non-linear thermal analysis is performed to calculate the temperature history of the entire domain. Then, the results of the thermal analysis are applied as a thermal body load in a non-linear mechanical analysis determining residual stress and distortion. The finite element (FE) models for both thermal and structural analysis are the same. The general-purposed FE program ANSYS is used for the analysis. A full Newton-Raphson iterative solution technique with direct sparse matrix solver is employed for obtaining a solution. During the thermal analysis, the temperature and the temperature dependent material properties alter very rapidly. Thus, the full Newton-Raphson technique with using modified material properties is believed to give more precise results.

A conventional technique named "Element Birth and Death" is used for modelling of the deposited weld. A complete FE model is generated in the beginning of the analysis. However, all elements representing the deposited weld except elements for the tack welds are deactivated by assigning them a very low stiffness. During the thermal analysis, all the nodes of deactivated elements (excluding those shared with the base metal) are also fixed at room temperature till the birth of the respective elements. Deactivated elements are reactivated sequentially when they come under the effect of the welding torch. Here, eight-noded-brick elements with linear shape functions are used in the FE modelling.

A meshing investigation is done to find the most proper mesh according to the mesh size and accuracy of the FE results. The basic mesh size is shown in Fig. 2, while some finer and coarser meshing models are studied and the results are compared with the experimental measurements. Selecting the most effective mesh size leads to the accurate results along with time-consuming calculations.

\section{EXPERIMENTAL PROCEDURES}

\subsection{Sample Description}

A pressure vessel is constructed from two $304 \mathrm{~L}$ stainless steel pipes and two standard caps while the diameter and thickness is equal to 5 inch and $6 \mathrm{~mm}$ respectively (Fig. 3). The pipes and caps are stress relieved at $450^{\circ} \mathrm{C}$ for 6 hours, before being employed in manufacturing process of pressure vessel. The welding processes include a main centre weld to join the pipes, welding process of right cap, left cap weld and nozzle welding according to the 
specifications mentioned in Table 1. The welding processes are semi-automated tungsten inert gas (TIG) welding except the nozzle which is manually welded. The pipes and caps are welded in V-groove $\left(60^{\circ}\right.$ included angle) while root gap is equal to $2 \mathrm{~mm}$ and $308 \mathrm{~L}$ is selected as the filler material. The temperature history is recorded by two thermocouples (type K) during the welding process of Cap 2. The thermocouples, shown in Fig. 4, are installed on the shell with $12.5 \mathrm{~mm}$ and $25 \mathrm{~mm}$ distance from the centre of cap weld. The recorded temperatures, shown in Fig. 5 , are compared with those obtained from the FE welding simulation to verify the thermal analysis results. All of the weld reinforcements are removed by $30000 \mathrm{rpm}$ hand grinder to facilitate ultrasonic measurements. However, temperature during the grinding process is kept less than $50^{\circ} \mathrm{C}$ to prevent generation of thermal stresses. The residual stresses are measured by the ultrasonic and hole-drilling method with the characterizations shown in Fig. 3.

\subsection{Measurement device}

The measurement device, shown in Fig. 6, includes an ultrasonic box, computer and time of flight (TOF) measuring element. The ultrasonic box is a portable ultrasonic testing device with sample rate of $100 \mathrm{MHz}$. The analogue to digital (A/D) converter is controlled by synchronization between the pulser signal and the internal clock. The clock works with the resolution of 1 ns which allows precise TOF measurement. The name of "ultrasonic box" is borrowed from its manufacturer while the reason for this name has been multi-functional capabilities along with small size of the box which made it a portable ultrasonic device. TOF measuring element includes three normal transducers assembled on an integrated wedge to measure the time of flight. A poly methyl methacrylate (PMMA) material, under the trademark Plexiglas, is cut by laser to construct the wedge. The TOF is measured on a scanning path (shown in Fig. 3) by two different types of wedge needed to measure hoop and axial stress separately. Surface curvature of pipe is taken into account in laser cutting of the bottom surface in both of the wedges.

A three-probe arrangement is utilized, with one sender and two receivers in order to decrease the environmental effects on the travel time. Accurate manufacturing along with proper assemblage of the experimental devices leads to precise TOF measurement though some of the environmental parameters (like changing temperature, humidity, ultrasonic couplant thickness and etc.) affect on TOF measurement inevitably. However, a thermocouple and thermometer are utilized to record the environmental temperature change to compensate its effect on the TOF but measuring and compensation of all the environmental effects is impractical. Hence, using three-probe configuration is recommended to decrease theses effects. In three-probe configuration, the TOF between first and second receiver is measured which is less sensitive to the environmental effects in comparison with the TOF measured between sender and receiver.

Twelve transducers in four different frequencies are used while their nominal frequencies are $1 \mathrm{MHz}, 2 \mathrm{MHz}, 4 \mathrm{MHz}$ and $5 \mathrm{MHz}$. Three normal transducers with the same frequency are assembled in each wedge where the diameter of the piezoelectric elements is $6 \mathrm{~mm}$. The pressure needed to keep the couplant film constant, is provided by a screw while a load cell is employed for load adjustment.

The scanning paths start from melted zone (MZ) of the right cap, pass the main centre weld and end to $\mathrm{MZ}$ of the left cap. Moving step is equal to $2 \mathrm{~mm}$ for the points near and on the MZ while it is increased to $10 \mathrm{~mm}$ far away the weld. The TOF is measured three times for each point and the average data is calculated. The path should be scanned for four times with four different frequencies of the transducers.

\subsection{Determination of $L_{C R}$ Penetration Depth}

When the $L_{C R}$ technique is applied to an application with limited wall thickness, the depth of the $L_{C R}$ wave penetration is expected to be a function of frequency. However, there is no definite relation between $L_{C R}$ depth and frequency. Hence, the $L_{C R}$ depth should be measured experimentally. A variable depth groove is cut in a pipe, with the same material and thickness of the pressure vessel, to produce a barrier to physically prevent the $L_{C R}$ wave from reaching the receiver transducer. It is found that a $1 \mathrm{~mm}$ depth groove could proportionally prevent a $5 \mathrm{MHz} L_{C R}$ wave to pass, which indicates that the penetration depth of such a $L_{C R}$ wave is $1 \mathrm{~mm}$. Similarly, the penetration depth of $4 \mathrm{MHz}, 2 \mathrm{MHz}$ and $1 \mathrm{MHz} L_{C R}$ wave is measured $1.5 \mathrm{~mm}, 3.5 \mathrm{~mm}$ and $6 \mathrm{~mm}$ respectively.

It should be noticed that the variable depth groove should be wide with rounded tip to prevent the diffraction from the groove tip which leads to inaccurate measuring of the penetration depth. For example, first groove was produced by using a milling tool with $2 \mathrm{~mm}$ diameter which the diffraction yielded to non logical results of 
penetration measurement but using another tool with $6 \mathrm{~mm}$ diameter solved the diffraction problem. The measured penetration depths much different from wavelength of the $L_{C R}$ wave is considered as the non logical results. Furthermore, this groove could proportionally (not completely) prevent passing the wave hence, it is recommended to interpret the noise equal to zero passing the wave. It means that, zero passing is occurred when amplitude of the $L_{C R}$ wave is equal to the ultrasonic noises and the related depth would be the measured penetration depth.

\subsection{Acoustoelastic constant evaluation}

To evaluate the acoustoelastic constant $(L)$, the pressure vessel is hydro tested according to the setup shown in Fig. 7. A thermal stress relieving is performed and the value $t_{0}$ is then measured directly on the parent material (PM), melted zone (MZ) and heat affected zone (HAZ) of the main and caps weld. The pressure vessel is filled with the water and the internal pressure is increased step by step via a hydraulic hand pump. The stress on the outer surface is calculated according to the ASME-Sec. VIII while, two strain gauges are employed to verify the calculations. The measurement process includes holding in a certain pressure; moving the TOF measuring element on the PM, HAZ and $\mathrm{MZ}$; measuring the TOF on different zones and measuring the strain by the strain gauges. All the procedures are repeated for eight different internal pressures by axial and hoop wedge. The acoustoelastic constant $(L)$ of the PM, $H A Z$ and $M Z$ is then calculated based on Eq. (1). However, the HAZ constant measurement is impractical in the axial direction because of dimensional limitations.

\section{RESULTS AND DISCUSSION}

The results of hydro test are shown in Fig. 8 and Fig. 9 corresponding to the hoop and axial direction respectively, where the slope of the lines represents $L$ acoustoelastic constants. Since the elastic modulus of the tested material is equal to $200 \mathrm{GPa}$ hence, the fractional change in travel time (vertical axes of Fig. 8) could be between 0 to $0.350 / 200$ during the hoop constant measurement. The value of measured hoop constant is $1.78,1.86$ and 2.248 in the $H A Z, P M$ and $M Z$ respectively which show that the HAZ constant is less than the PM whereas the maximum is the $\mathrm{MZ}$ constant. There is some difference between hoop constant with the axial constant which is measured equal to 2.09 and 2.28 in the PM and MZ respectively. However, the axial constant of the HAZ is impractical to be measured because the width of HAZ is less than $2 \mathrm{~mm}$ which is not enough to distinguish between $\mathrm{HAZ}$ and $\mathrm{MZ}$ measurements in axial direction.

In this study, a 3D finite element analysis is used to predict residual stresses through the pressure vessel thickness. The results of the finite element method are shown in Fig. 10 and Fig. 11 for different depths corresponds to the measured penetration depth of the ultrasonic transducers. The hoop residual stress is tensile in centre of the main and caps weld whereas it converts to compressive stress near the HAZ. The hoop stress behaviour is the same for inner and outer surface of the pressure vessel despite the fact that the axial stress considerably differs through the thickness. The hoop stress changes from 107.57 MPa to 136.25 MPa by moving from the outer to inner surface while the axial stress converts from -221.41 MPa to $266.59 \mathrm{MPa}$ in centre of the main weld. Furthermore, all the results demonstrate higher residual stress for the main weld in comparison with the caps weld. However, stress level of the Cap 2 is higher than those calculated for the Cap 1.

It should be noted that ultrasonic method measures the average of stresses in determined depths. For example, the $1 \mathrm{MHz} L_{C R}$ wave travels in $6 \mathrm{~mm}$ beneath the surface and gives the average of residual stress in this zone. Therefore, the FE residual stresses for all the nodes located in $6 \mathrm{~mm}$ under the surface are extracted and their averages are calculated to compare with measurements performed by the $1 \mathrm{MHz} L_{C R}$ wave. It means that, the results shown in Fig. 10 and Fig. 11 cannot be compared with the ultrasonic results because they are exact results. Therefore, the averages of FE residual stresses are calculated and shown in Fig. 12 for the main weld. Comparing these results with those shown in Fig. 11, demonstrates notable difference in the stress behaviour. The considerable difference between the axial stress in the inner and outer surface, cannot be observed in case of average results. In addition to qualitative change, the value of hoop and axial stress is also differed, e.g. the axial stress is altered from $266.59 \mathrm{MPa}$ (exact result corresponds to inner surface) to $-30.85 \mathrm{MPa}$ (average result corresponds to $6 \mathrm{~mm}$ ). It means that the tensile axial stress of the inner surface cannot be evaluated by the ultrasonic inspection from the outer surface.

To compare FE results with ultrasonic measurements, it is needed to verify the FE model with the experimental results. The validation of the thermal analysis is done by comparison of the temperature history recorded by the thermocouples, shown in Fig. 5, and those estimated by FE analysis. The mechanical analysis is also verified by hole- 
drilling method according to Fig. 13. The average results of FE residual stress in $2 \mathrm{~mm}$ depth are compared with those of hole-drilling method because this method also gives the average of residual stress measured along the 2 $\mathrm{mm}$ depth hole. The hole-drilling method is performed in five different points based on the characterizations described in ASTM-E837. The comparison between thermal and mechanical analysis with the experimental results shows good agreement which validates the FE welding simulation.

The results of ultrasonic measurements are shown in Fig. 14-Fig. 17 according to the frequency of ultrasonic transducer used to measure the TOF. All of the results of ultrasonic measurements are compared with finite element analysis showing an acceptable agreement. The disagreement between FE and ultrasonic, listed in Table 2, is differed according to the ultrasonic test frequency and tested zone.

According to the results of Table 2, the ultrasonic accuracy in axial direction is higher than the hoop stress measurements which can be justified by surface distortions of the pressure vessel. The positioning of the hoop wedge is more difficult than the axial wedge because of welding deformations of tested surface. However, the measurement error of axial stress is increased in the HAZ because the acoustoelastic constant is not measured in this zone.

Comparison between different test frequencies shows that the $L_{C R}$ waves work more accurately by employing the lower frequency transducers. It means that the disagreements between the FE and ultrasonic measurement performed by $5 \mathrm{MHz}$ transducer is higher than those obtained by $4 \mathrm{MHz}$ and the minimum occurs at $1 \mathrm{MHz}$. Higher error of higher frequencies can be justified by the fact that the low frequency transducers produce sharp and more powerful echo in the receiver than high frequency transducers. Therefore it is necessary to increase the "Gain" by using higher frequencies which will lead to less resolution and higher measuring error in the TOF.

However, the disagreement between the FE and ultrasonic results do not exceed than $\pm 40 \mathrm{MPa}$ which confirms the capability of $F E L_{C R}$ method (which is the combination of finite element welding simulation and ultrasonic stress measurement by $L_{C R}$ waves) to evaluate welding residual stress through thickness of the pressure vessel.

\section{CONCLUSION}

The main goal of this paper is ultrasonic evaluation of hoop and axial residual stresses in a stainless steel pressure vessel through the thickness. The ultrasonic measurements are compared with residual stresses obtained from a FE analysis validated by hole-drilling method. According to the achieved results, it can be concluded that:

1- The hoop residual stress is tensile in the weld centreline while the axial stress is notably differs through the thickness of the vessel.

2- The $L_{C R}$ ultrasonic method measures the average of stresses in determined penetration depth of transducers. Therefore, if measuring the stress in an exact depth is needed, the ultrasonic method is not recommended.

3- The tensile axial stress of the vessel inner surface cannot be evaluated by the ultrasonic inspection from the outer surface.

4- Distribution of the axial residual stress is more uniform than hoop stress which can be justified by surface distortions of the pressure vessel.

5- The acoustoelastic constant of the HAZ cannot be measured in the axial direction which leads to higher measurement error of the axial stress results.

6- The average results of FE residual stress in $2 \mathrm{~mm}$ from the surface are in good agreement with those of hole-drilling method.

7- Ultrasonic measurements of hoop and axial residual stresses are compared with finite element analysis and show an acceptable agreement.

8- The disagreement between the FE and ultrasonic results is increased by using high frequency transducers.

9- The welding residual stress through the thickness of stainless steel pressure vessel can be evaluated by using the $F E L_{C R}$ method. 


\section{REFERENCES}

[1] Thompson R B, Lu W Y, Clark Jr A V. Handbook of Measurement of Residual Stress. In: Lu J, James M, Roy G, editors. Society for Experimental Stress Analysis, Bethel, Connecticut; 1996, p. 149-78.

[2] Schneider E. Structural and Residual Stress Analysis by Nondestructive Methods. In: Hauk V, editors. Elsevier, Amsterdam; 1997, p. 522-63.

[3] Santos A, Bray D E. Ultrasonic Stress Measurement Using PC Based and Commercial Flaw Detectors. Rev Sci Instrum 2000; 71:3464-9.

[4] Santos A A, Bray D E. Application of Longitudinal Critically Refracted Waves to Evaluate Stresses in Railroad Wheels. In: ASNT Spring Conference, Birmingham, Alabama 27-31 March, Vol. 5; 2000.

[5] Bray D E, Chance B. Practical Aspects of Ultrasonic Stress Measurement. In: Darvennes C, editors. ASME NDE-Vol. 19; 1999, p. 75-9.

[6] Tanala E, Bourse G, Fremoit M, De Belleval J F. Determination of Near Surface Residual Stresses on Welded Joints Using Ultrasonic Methods. NDT \& E Int 1995; 28:83-8.

[7] Szelazek J. Monitoring of Thermal Stresses in Continuously Welded Rails with Ultrasonic Technique. In: ECNDT, Copenhagen 26-29 May, Vol. 3, No. 6; 1998.

[8] Bray Don E. Ultrasonic Stress Measurement and Material Characterization in Pressure Vessels, Piping, and Welds. Journal of Pressure Vessel Technology 2002; 124:326-35.

[9] Rossini N S, Dassisti M, Benyounis K Y, Olabi A G. Methods of measuring residual stresses in components. J Materials \& Design 2012; 35:572-88.

[10] Egle D M, Bray D E. Measurement of Acoustoelastic and Third-Order Elastic Constants for Rail Steel. J Acoust Soc Am 1976; 60(3):741-4.

[11] Goldak J, Akhlaghi M. Computational Welding Mechanics. Springer; 2005.

[12] Lindgren LE. Finite element modelling and simulation of welding part 2: improved material modeling. J Thermal Stress 2001; 24:195-231.

[13] Zhu X K, Chao Y J. Numerical simulation of transient temperature and residual stresses in friction stir welding of 304L stainless steel. Journal of Materials Processing Technology 2004; 146: 263-72. 
8. LIST OF TABLE CAPTIONS

Table 1: Welding specifications of Pressure Vessel

Table 2. Disagreement between FE and ultrasonic results 


\section{9. $\quad$ LIST OF FIGURE CAPTIONS}

Fig. 1. $L_{C R}$ Wave Propagated in the Stainless Steel Pipe through the PMMA Wedge

Fig. 2. Basic FE model of the Pressure Vessel

Fig. 3. Pressure Vessel Dimensions

Fig. 4. Thermocouple position

Fig. 5. Temperature history recorded by Thermocouples

Fig. 6. Measurement Devices for TOF measurement

Fig. 7. Hydro test setup to measure acoustoelastic constant of the pressure vessel

Fig. 8. Result of hydro test to evaluate hoop acoustoelastic constant

Fig. 9. Result of hydro test to evaluate the axial acoustoelastic constant

Fig. 10. Hoop Residual Stresses obtained from FE analysis for a) entire of the pressure vessel and b) main weld

Fig. 11. Axial Residual Stresses obtained from FE analysis for a) entire of the pressure vessel and b) main weld

Fig. 12. Average of a) Hoop and b) Axial Residual Stresses obtained from FE analysis for the Main Weld

Fig. 13. Comparison of FE and Hole-Drilling results corresponding to the a) Hoop and b) Axial Residual Stresses

Fig. 14. Comparison of FE and Ultrasonic results performed by $5 \mathrm{MHz}$ Transducer corresponding to the a) Hoop and b) Axial Residual Stresses

Fig. 15. Comparison of FE and Ultrasonic results performed by $4 \mathrm{MHz}$ Transducer corresponding to the a) Hoop and b) Axial Residual Stresses

Fig. 16. Comparison of FE and Ultrasonic results performed by $2 \mathrm{MHz}$ Transducer corresponding to the a) Hoop and b) Axial Residual Stresses

Fig. 17. Comparison of FE and Ultrasonic results performed by $1 \mathrm{MHz}$ Transducer corresponding to the a) Hoop and b) Axial Residual Stresses 